\title{
Turrell's tunnelling
}

Turner delights his audiences with his magical depictions of nature on canvas. James Turrell is using nature itself as the canvas - by digging into a volcanic crater to create a new kind of observatory.

\section{Martin Kemp}

$\mathrm{T}$ urner, the magician of light and colour who was the dominant figure in British Romantic painting in the first half of the nineteenth century, dealt less with conventional boxes of pictorial space than with "the wide concave of the circumambient air". His phrase should be understood in the context of his intuition that "the building of Nature" was "too colossal for the intellectual capacity, its height to measure or its depth to fathom - the Universe and Infinitude".

Turner's visual resources were limited to the traditional medium of paint on flat surfaces - if 'limited' is the right word for a painter whose floating visions of space, light and colour suggest more in the way of "Infinitude" than seems possible.

\section{By contrast, the American artist James} Turrell is creating spatial structures on a huge scale to sculpt the passage of natural light and even to reshape the sky itself - in a Turnerian quest to stretch out to infinite reaches of time and space.

In 1975, Turrell searched for a suitable site for the realization of his vast vision of a new "Skyspace". An aerial survey of the western United States drew his attention to Roden Crater, a cinder cone at the east of the San Francisco volcanic plateau. The project started there in 1979 and is due to be completed early in the next millennium. It involves the excavation of geometrical spaces that will serve as exploratories for our perceptions, as vessels for the enclosure of solar, lunar and stellar illumination, as devices that inscribe time, like a sundial or gnomon, and as a compound observatory in which precision, wonder and imagination are united.

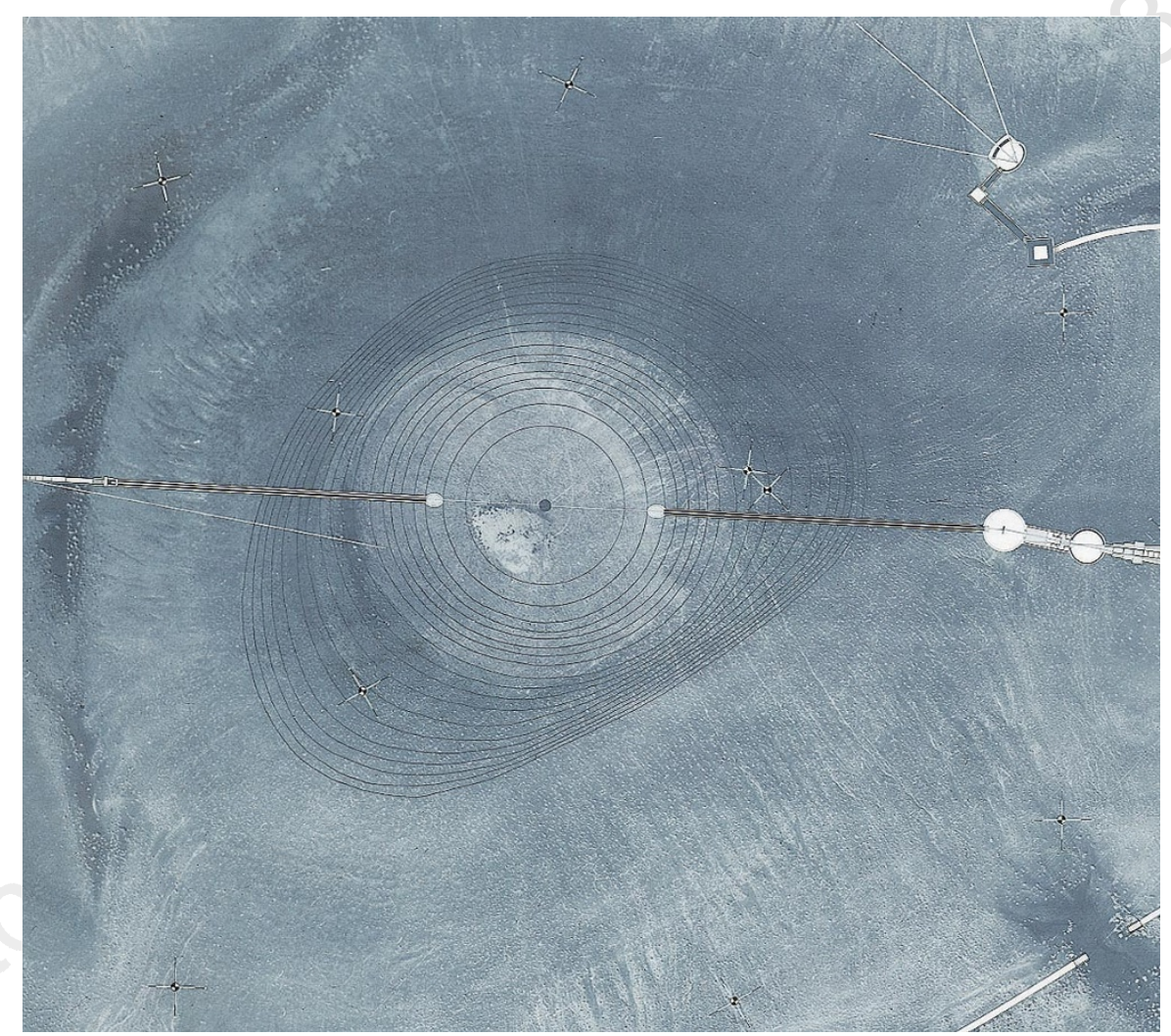

James Turrell's Roden Crater - Negative Site Plan, 1992 (below) and detail of crater (above).

At the centre is the crater transformed into a bowl with an even rim, 3,000 feet in diameter. For the observer low in the bowl, the vault of the heavens is transformed. Subjectively perceived as a shallow dome - a phenomenon much debated in psychology - the sky appears to be anchored circumferentially to the crater's rim. Around the crater, chambers are being tunnelled out in such a way that the passage of light — night into day - will paint the interiors with a succession of shaping shadows and luminous colours.

The fumarole, or secondary vent, will be

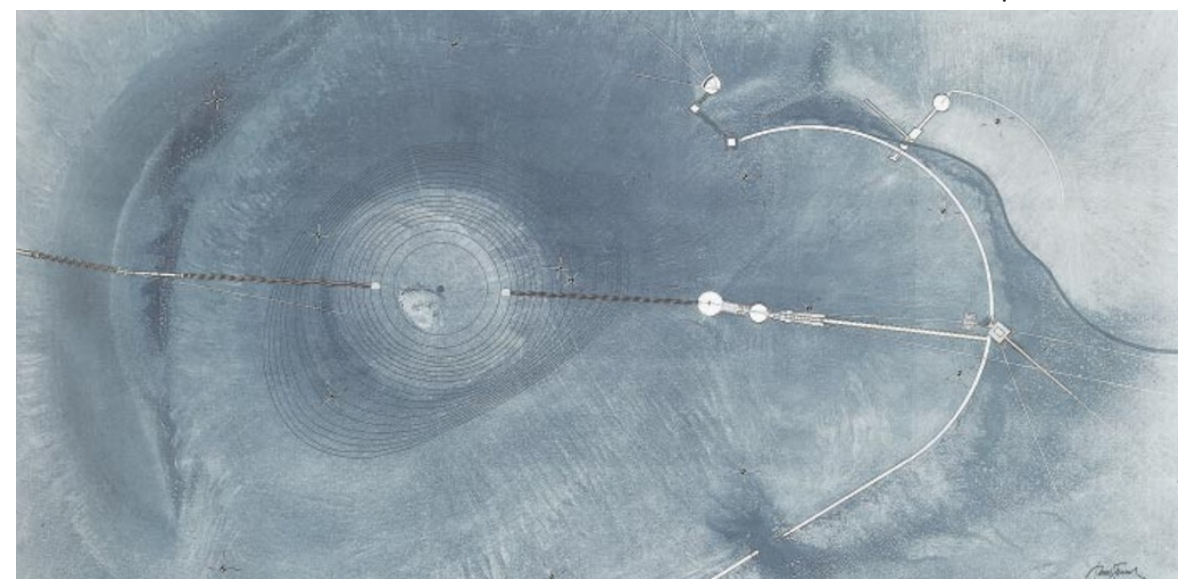

the focus for chambers oriented towards the four compass points, joined to a series of linked interiors to provide varying spatial perceptions, and a tunnel 1,035 feet long leading into an oval sky chamber as an atrium for the main bowl. The viewer's subsequent progress to the top of the rim culminates in the reattachment of the dome of the sky to the far horizon of the land.

Turrell's project is presented through models, paintings, drawings, prints and photo-works. Its resonances are astonishingly rich. They range from the prehistoric observatories of stone circles and ancient temples, through the great astronomical castle and garden of Tycho Brahe on the Danish island of Hven, and the camera obscuras of Jesuit magicians in the seventeenth century, to the huge dishes of modern observatories.

At the project's heart is a vision worthy of Turner: "looking at light in a Skyspace is akin to a worldless thought" — and such a thought has boundless potential.

Martin Kemp is in the Department of the History of Art, University of Oxford, 35 Beaumont Street, Oxford OX1 2PG, UK. 